
\title{
25 Research Square \\ COVID 19 Vaccine Hesitancy: A Protocol for Systematic Review and Meta-Analysis
}

\section{Emmanuel Okechukwu Nna ( $\nabla$ e.nna@themping.org )}

The Molecular Pathology Institute, Enugu https://orcid.org/0000-0001-6791-2336

\section{Michael Abel Alao}

University College Hospital Ibadan

\section{Babatunde Ogunbosi}

University College Hospital Ibadan

\section{Uchenna Okeke}

Nigerian Navy Reference Hospital Calabar

\section{Canice Anyachukwu}

University of Nigeria Faculty of Health Sciences and Technology

\section{Protocol}

Keywords: hesitancy, COVID 19, vaccine hesitancy, COVID 19 vaccine, SARS COV-2, pandemic

Posted Date: January 20th, 2021

DOI: https://doi.org/10.21203/rs.3.rs-150209/v1

License: (c) (1) This work is licensed under a Creative Commons Attribution 4.0 International License. Read Full License 


\section{Abstract}

\section{Background}

The unprecedented development of COVID 19 vaccine within a few months and its introduction into the population brought a sigh of relief with the promise of preventing primary infections, halting spread, reducing hospitalization of infected people, and ultimately inducing herd immunity. However, public perception in many communities leaves a lot to worry about as the so much sort-after vaccine has been met with safety concerns, scepticism and hesitancy. We seek to produce a protocol for a reliable and accurate systematic review and meta-analysis on the hesitancy of COVID 19 vaccine uptake in the mist of a global pandemic.

\section{Methods}

We developed a search strategy using MeSH terms, text words and entry terms. Nine databases will be searched: PubMed, Embase, CINAHL, AJOL, Google Scholar, Web of Science, Cochrane Library, Research gate and Scopus. Only observational studies retrievable in the English Language will be included. The primary measurable outcome is the prevalence of COVID 19 vaccine hesitancy globally. The secondary outcomes are factors that influence COVID 19 vaccine hesitancy including race, age, gender, occupation, socioeconomic class, level of education, geographic location, misinformation, social media influence and vaccine safety. Identified studies will be screened, deduplicated, selected and data items extracted using DistillerSR software. All studies will be assessed for methodological, clinical and statistical heterogeneity. Assessment of risk of bias in the selected studies will be performed using the NIH Quality assessment tool for observational studies and the Cochrane tool of risk of bias. Publication bias will be assessed using the funnel plot as well as Egger's regression intercept. The pooled prevalence, standard error and $95 \% \mathrm{Cl}$ will be reported. The strength of evidence from this analysis will be assessed by using NIH Quality Assessment for Systematic Reviews and Meta-analysis.

\section{Discussion}

The outcome of this analysis will give insight into the level of COVID 19 vaccine hesitancy and its correlates across geographical regions globally. It will examine if herd immunity via vaccination is attainable at the pooled rate of hesitancy. This will help Governments to redesign their public messages and sensitization.

Systematic Review Registration

This protocol has been registered with PROSPERO, registration number CRD42021231165.

\section{Background}

The COVID 19 pandemic has resulted in an unprecedented humanitarian and economic crisis far beyond what could best be imagined. $(1,2)$ The rapidity and intensity with which the infection spread across the 
globe reminded everyone and all major global players that the world was not as big as we thought. The world is rather a small village where our unified effort is required to sustain the existence. $(1,3,4)$ As of January 23, 2021, COVID 19 has infected 90.3 million individuals with 2 million deaths reported. $(4,5)$.

Although Governments, not for profit organizations and private individuals have invested huge resources on identifying a novel medical cure for COVID 19, the prospect thus far is way off from the desirable outcome.(6-8) The WHO and other international bodies have championed several interventions including infection and prevention control measures which include regular and proper hand-washing, respiratory hygiene and etiquette, events and meeting arraignment rules and use of adequate personal protective equipment by health workers. $(9,10)$ These interventions have substantially reduced the spread of the infection, consequently attenuating the curve of the pandemic. However, the results are short-lived as the magnitude of the pandemic still remains a global public health emergency.(11) More disturbing is the emergence of viral mutants that are highly capable of rapid spread and infectivity. These mutants have triggered second waves in most countries, fueling more deaths, panic and losses.

The most visible approach is to develop a long-term intervention targeting primary prevention. As a rapid response to the urgent need for a long-term solution to curb COVID 19 infection, several novel vaccines were developed, all driven by the private sector with enormous government support. Many of the targeted vaccines have passed phase 3 clinical trial and have been introduced into the population while some are still being investigated. $(12,13)$ Many of the approved vaccines were given emergency use authorization. $(14,15)$ However, public perception in many communities leaves a lot to worry about as the so much sortafter vaccines have been met with safety concerns, scepticism and hesitancy.(16, 17) There are concerns regarding the safety of the vaccine, mechanism of immune induction, the complexity of maintaining a cold chain giving the tight storage temperature requirement especially in low resource settings, and lots of misinformation, conspiracy theories, information gap and superstitions to mention a few. $(18,19)$

Evidences abound to the efficacy of immunization as one of the most effective intervention in human history. $(20,21)$ Currently the vaccines are designed to help reduce the increasing levels of hospitalization, fatalities, infections and to help achieve herd immunity (achieving exposure of greater than $70 \%$ in all populations). Most of the approved vaccines have reported greater than $90 \%$ efficacy in the phase 3 trials. $(22,23)$ The three notable vaccines for global use are the BioNtech-Pfizer, Moderna and Astrazeneca. $(12,23)$ Other vaccines include the Chinese vaccine, the Russian Sputnik vaccine and Indian vaccine. $(24,25)$

The success of achieving herd immunity in the community depends not only on the production and availability of vaccines but also the uptake.(26) How many people are willing to take the vaccine in a community ? At present, available data on COVID 19 vaccine hesitancy is largely from observational studies with conflicting reports, between $22-78 \%$ hesitancy levels reported.(19, 27) This varies across races, religious inclinations, locations and socio-economic class. $(28,29)$ To produce a robust effective evidence towards promoting mass vaccination, a systematic review and meta-analysis will help to consistently and accurately delineate the various determinants of hesitancy for COVID 19 vaccine uptate. 
Many of the earlier interventions at the onset of the pandemic worked poorly in many countries.(30) This has fueled the fears driving hesitancy. We aim to produce a protocol to enable a reliable and accurate systematic review and meta-analysis for global COVID 19 hesistancy.

\section{Methods And Design}

Objective:

The main objective of this study is to determine the pooled hesitancy rate for COVID 19 vaccine uptake globally.

\section{Review Questions:}

1. What are proportions of people who are hesitant to take COVID 19 vaccine globally?

2. How does race, religion, location, occupation, socioeconomic class, level of education and gender influence COVID 19 hesitancy globally?

3. How does misinformation and lack of information influence COVID 19 hesitancy globally?

4. How does social media influence COVID 19 vaccine hesitancy?

5. How does safety concerns and adverse events influence COVID 19 vaccine hesitancy?

6. With the pooled hesistancy rate globally, is it possible to achieve herd immunity by vaccination?

\section{Study Characteristics:}

\section{Design}

This is a protocol for systematic review and meta-analysis of obervational studies from 2020 to present time. It is designed to enable a robust, reliable and accurate data synthesis on COVID 19 hesitancy qualitatively and quantitatively.

\section{INCLUSION CRITERIA:}

1. Observational studies: Cohort studies, case controls, cross-sectional studies, historic cohort studies.

2. Studies must report the primary outcome: COVID 19 vaccine hesitancy.

3. Study must be retrievable in the English language.

\section{Exclusion criteria:}

a) Reviews, editorials, interventional studies, commentaries, methodological articles, letters to editors, case reports

b) Duplicates/ replicates of studies.

c) Studies not retrievable in the English Language. 
PICOS

Populations: global population that are eligible for COVID 19 vaccination

Intervention: COVID 19 vaccine

Comparator: Willingness to take the COVID 19 vaccine

Outcomes: The primary outcome is proportion of people globally who are hesitant to take COVID 19 vaccine.

The secondary outcomes include categorical and quantitative variables that influence hesitancy to COVID 19 vaccination. These variables include race, occupation, gender, location, religion, socioeconomic class and level of education. It also includes variables such as genuine safety concerns, levels of misinformation and social media influence.

\section{Information sources}

The search will employ sensitive topic-based strategies designed for each database. The search will be carried out in the following databases: PUBMED, EMBASE, CINAHL, RESEARCHGATE, AJOL, GOOGLE SCHOLAR, WEB OF SCIENCE, SCOPUS and COCHRANE LIBRARY. Only observational studies will be included, from 2020 to present time.

\section{Search strategy}

The search strategy includes MESH terms, text words and entry terms. Table 1 shows the search strategies as used in the Pubmed. The same search strategy will be used in other databases with slight modifications.

\section{Data Extraction and Management}

\section{a. Data Extraction}

Data will be managed in three main softwares: DistillerSR, CMA version 3 and Microsoft Excel.

Identified studies will be screened independently in pairs and blindly using the DistillerSR software at 6 different levels:

i. Level 1 would involve screening of identified studies for the study design. Only observational studies would be accepted

ii. Level 2 will involve screening of identified studies in the titles and abstracts using entry terms, keywords and meSH terms

iii. Level 3 will involve further screening of the contents of articles by reading the full text articles using the same search strategy. 
iv. Level 4 will involve snowballing of literature on references from included studies.

v. Level 5: Studies will be screened at outcome levels to select those that reported the primary outcome with or without secondary outcomes

vi. Level 6 will involve grey literature that report primary outcome and or secondary outcomes.

Conflicts during screening will be resolved by a third independent reviewer who servies as a tie breaker.

\section{b. Selection Process:}

Screened studies will be selected based on study charateristics: study design, inclusion/exclusion criteria and agreement between two independent and blinded reviewers. Authors of included studies with missing data will be contacted via email and telephone. After selection, studies will be deduplicated. Data items wil be extracted from selected studies into predefined forms in the DistillerSR.

c. Data Collection: Data items to be extracted from selected studies include:

i. Surname of first author and year of publication

ii. Prevalence of COVID 19 hesitancy.

iii. Race, Religion, Location, Occupation

iv. Socioeconomic class, Gender, Level of Education

v. Vaccine safety and adverse events

vi. Level of misinformation, social media influence and information gap.

Data items will be exported into predefined format in Microsoft Excel, to be imported into the CMA software for quantitative analysis.

The effect size for the primary outcome is prevalence. The effect sizes for secondary outcomes are some categorical and others quantitative.

\section{Data synthesis}

Extacted data items will be used for both narrative synthesis and quantitative analysis.

The following criteria will be applied for analysis:

a. Studies that passed the methodological quality assessment using the NIH quality assessment tool will be crosschecked with the Cochrane Risk of Bias tool will be included. The results will be presented in tabular format, indicating all the extractable data items as listed under data collection.

b. All studies with primary outcomes will be used for narrative synthesis. 
c. All studies with primary outcomes and secondary outcomes that pass heterogeneity tests will be used for quantitative synthesis.

d. Further Analysis: Subgroup analysis will be performed using variables such as race, gender, socioenomic status, geographical location (country), level of education and occupation. Metaregression will be performed on quantitative variables such as level of misinformation (\%), level of safety concerns (\%), level of influence by social media (\%) and age.

e. Where heterogeneity exists, sensitivity testing using include/exclude functions in the CMA software will be performed.

f. The computational model for analysis is Random effect model since the several studies across the globe will be included.

\section{Risk of bias}

The risk of bias in the included studies will be assessed for the individual article using the National Institute of Health $(\mathrm{NIH})$ Quality assessment tool for observational cohort and cross-sectional studies. The NIH Quality assessment tool has 14 questions, with scores about 8 indicating good quality study. This will be cross-checked with the Cochrane tool of risk of bias assessment for non-randomized study. Publication bias in the selection of studies will be visually assessed using the funnel plot (trim and fill method) and test for asymmetry. Other statistical tests such as Egger's regression intercept, Begg and Mazumdar's rank correlation and Orwin's fail-safe $\mathrm{N}$ will be used where appropriate. Studies with extreme bias will be subjected to sensitivity testing using the include/exclude function in the CMA Software.

\section{Assessment of Meta-bias}

Meta-bias will be assessed as follows:

i. Method of testing/reporting of COVID 19 hesitancy at outcome level.

ii. Index of reporting outcomes in studies: Studies that were reported in different indices but similar in outcome and design will be converted to the primary effect size (prevalence) based on individual case evaluation.

iii. Heterogeneity will be assessed at the study level using the Q statistics, and its $p$-value, $I^{2}, \mathbb{\Xi}^{2}$ (Tau squared). As a rule of thumb, $I^{2}$ values of less than $40 \%$ will be considered low heterogeneity while values $>40$ but $<75 \%$ will be considered moderate and values $>75 \%$ are high.

\section{Presentation and Reporting of Results}

The study selection process will be summarised in a Prisma flow chart according to the PRISMA 2015 Statement and PRISMA-P Checklist. A table of the search strategy in various databases showing text words, MeSH terms, and entry terms will be included. A list of included studies with extracted data items will be summarized in a table. Quantitative data such as prevalence of COVID 19 hesitancy, $95 \% \mathrm{Cl}, \mathrm{P}$ values, and relative weights assigned to studies and heterogeneity tests will be included in the forest 
plots. A table of quality scores and risk of bias of each eligible study will be included. Forest and regression plots to show sub-group analysis and meta-regression respectively will be included.

\section{Discussion}

This study does not require ethical approval for dissemination. The outcome of this analysis will give insight into the level of COVID 19 vaccine hesitancy and its correlates across geographical regions globally. The study will explore the possibility of reaching global herd immunity based on the pooled prevalence of COVID 19 hesitancy, if vaccination alone is relied upon. This will help Public health Institutions and Governments across the World to calibrate their public messages and sensitization approaches. The outcome of this study will be published in peer-reviewed scientific journals. The overall strength of evidence of this study will be assessed by the NIH Quality Assessment for Systematic Reviews and Meta-analysis.

\section{Abbreviations}

GRADE: Grades of Recommendation, Assessment, Development and Evaluation.

PRISMA-P: Preferred Reporting Items for Systematic reviews and NIH: National Institute of Health CMA: Comprehensive Meta-Analysis Software

PROSPERO: International Prospective Register for Systematic Reviews

\section{Declarations}

\section{Ethics and Dissemination}

Ethical approval will not be required, since this study will rely solely on secondary source of data.

\section{Contributions}

EN conceived the project. AMA and EN designed the study; EN, AMA, BO, $U O$ and CA did searches on PubMed and other databases. AMA started the early draft, EN expanded the draft fully and all authors corrected the manuscript and consented to publication.

\section{Funding}

The study is self-funded by the authors.

\section{Support}

The Molecular Pathology Institute provided access to DistillerSR via the subscription. 
Guarantor of the Review: Dr Emmanuel Nna

\section{Ethical Approval / Consent to participate:}

Not applicable

Informed Consent: Not applicable

\section{Availability of data and material}

Data and material from this study will be made available to the public unhindered.

\section{Competing Interest:}

The author(s) declared no potential conflicts of interest with respect to the research, authorship, and/or publication of this article.

\section{Acknowledgements}

We thank the staff of the Molecular Pathology Institute for assistance.

\section{Ammendments}

Important protocol amendments post registration will be recorded and included in dissemination.

\section{References}

1. WHO, 2020. A year without precedent: WHO's COVID-19 response. Accessed 11/01/2021 from https://www.who.int/news-room/spotlight/a-year-without-precedent-who-s-covid-19-response.

2. Harris A, Jones M. COVID 19-school leadership in disruptive times. Taylor \& Francis; 2020.

3. Sohrabi C, Alsafi Z, O'Neill N, Khan M, Kerwan A, Al-Jabir A, et al. World Health Organization declares global emergency: A review of the 2019 novel coronavirus (COVID-19). International Journal of Surgery. 2020.

4. World Health Organization, Coronavirus Disease 2019 (COVID-19): Situation Report, World Health Organization, Geneva, Switzerland, 2020.

5. World Health Organisation. Coronavirus disease (COVID-19) Weekly Epidemiological Update and Weekly Operational Update. Accessed 11///01/2021 from https://www.who.int/emergencies/diseases/novel-coronavirus-2019/situation-reports.

6. . !!! INVALID CITATION !!! $(6,7)$.

7. Adhikari SP, Meng S, Wu Y-J, Mao Y-P, Ye R-X, Wang Q-Z, et al. Epidemiology, causes, clinical manifestation and diagnosis, prevention and control of coronavirus disease (COVID-19) during the early outbreak period: a scoping review. Infectious diseases of poverty. 2020;9(1):1-12. 
8. Cascella M, Rajnik M, Cuomo A, Dulebohn SC, Di Napoli R. Features, evaluation and treatment coronavirus (COVID-19). Statpearls [internet]: StatPearls Publishing; 2020.

9. Guan W-j, Chen R-c, Zhong N-s. Strategies for the prevention and management of coronavirus disease 2019. Eur Respiratory Soc; 2020.

10. Razai MS, Doerholt K, Ladhani S, Oakeshott P. Coronavirus disease 2019 (covid-19): a guide for UK GPs. BMJ. 2020;368.

11. Xu S, Li Y. Beware of the second wave of COVID-19. The Lancet. 2020;395(10233):1321-2.

12. Mahase E. Covid-19: Pfizer and BioNTech submit vaccine for US authorisation. British Medical Journal Publishing Group; 2020.

13. Le TT, Andreadakis Z, Kumar A, Roman RG, Tollefsen S, Saville M, et al. The COVID-19 vaccine development landscape. Nat Rev Drug Discov. 2020;19(5):305-6.

14. Thomson K, Nachlis H. Emergency use authorizations during the COVID-19 pandemic: lessons from hydroxychloroquine for vaccine authorization and approval. Jama. 2020;324(13):1282-3.

15. Krause PR, Gruber MF. Emergency use authorization of Covid vaccines-safety and efficacy follow-up considerations. New England Journal of Medicine. 2020;383(19):e107.

16. Palamenghi L, Barello S, Boccia S, Graffigna G. Mistrust in biomedical research and vaccine hesitancy: the forefront challenge in the battle against COVID-19 in Italy. European journal of epidemiology. 2020;35(8):785-8.

17. Maglione MA, Das L, Raaen L, Smith A, Chari R, Newberry S, et al. Safety of vaccines used for routine immunization of US children: a systematic review. Pediatrics. 2014;134(2):325-37.

18. Grech V, Gauci C, Agius S. Vaccine hesitancy among Maltese healthcare workers toward influenza and novel COVID-19 vaccination. Early human development. 2020:105213.

19. Dror AA, Eisenbach N, Taiber S, Morozov NG, Mizrachi M, Zigron A, et al. Vaccine hesitancy: the next challenge in the fight against COVID-19. European journal of epidemiology. 2020;35(8):775-9.

20. Roush SW, Murphy TV, Group V-PDTW. Historical comparisons of morbidity and mortality for vaccine-preventable diseases in the United States. Jama. 2007;298(18):2155-63.

21. Jaca A, Mathebula L, Iweze A, Pienaar E, Wiysonge CS. A systematic review of strategies for reducing missed opportunities for vaccination. Vaccine. 2018;36(21):2921-7.

22. Mahase E. Covid-19: Vaccine candidate may be more than $90 \%$ effective, interim results indicate. British Medical Journal Publishing Group; 2020.

23. Knoll MD, Wonodi C. Oxford-AstraZeneca COVID-19 vaccine efficacy. The Lancet. 2020.

24. Poland GA, Ovsyannikova IG, Kennedy RB. SARS-CoV-2 immunity: review and applications to phase 3 vaccine candidates. The Lancet. 2020.

25. So AD, Woo J. Reserving coronavirus disease 2019 vaccines for global access: cross sectional analysis. bmj. 2020;371.

26. Omer SB, Salmon DA, Orenstein WA, Dehart MP, Halsey N. Vaccine refusal, mandatory immunization, and the risks of vaccine-preventable diseases. New England Journal of Medicine. 
2009;360(19):1981-8.

27. Salali GD, Uysal MS. COVID-19 vaccine hesitancy is associated with beliefs on the origin of the novel coronavirus in the UK and Turkey. Psychological medicine. 2020:1-3.

28. Callaghan T, Moghtaderi A, Lueck JA, Hotez PJ, Strych U, Dor A, et al. Correlates and disparities of COVID-19 vaccine hesitancy. Available at SSRN 3667971. 2020.

29. Barello S, Nania T, Dellafiore F, Graffigna G, Caruso R. 'Vaccine hesitancy'among university students in Italy during the COVID-19 pandemic. European journal of epidemiology. 2020;35(8):781-3.

30. Chowdhury R, Heng K, Shawon MSR, Goh G, Okonofua D, Ochoa-Rosales C, et al. Dynamic interventions to control COVID-19 pandemic: a multivariate prediction modelling study comparing 16 worldwide countries. European journal of epidemiology. 2020;35(5):389-99.

\section{Tables}

Table 1: Search strategy in PubMed

(("vaccines" [MeSH Terms] OR vaccine[Text Word]) OR (COVID 19 Vaccines Vaccines, COVID-19 COVID-19 Virus Vaccines COVID 19 Virus Vaccines Vaccines, COVID-19 Virus Virus Vaccines, COVID-19 COVID-19 Virus Vaccine COVID 19 Virus Vaccine Vaccine, COVID-19 Virus Virus Vaccine, COVID-19 COVID19 Virus Vaccines Vaccines, COVID19 Virus Virus Vaccines, COVID19 COVID19 Virus Vaccine Vaccine, COVID19 Virus Virus Vaccine, COVID19 COVID19 Vaccines Vaccines, COVID19 COVID19 Vaccine Vaccine, COVID19 SARS-CoV-2 Vaccines SARS CoV 2 Vaccines Vaccines, SARS-CoV-2 SARS-CoV-2 Vaccine SARS CoV 2 Vaccine Vaccine, SARS-CoV-2 SARS2 Vaccines Vaccines, SARS2 COVID-19 Vaccine Vaccine, COVID-19 Coronavirus Disease 2019 Vaccines Coronavirus Disease 2019 Vaccine Coronavirus Disease 2019 Virus Vaccine Coronavirus Disease 2019 Virus Vaccines Coronavirus Disease-19 Vaccines Coronavirus Disease 19 Vaccines Vaccines, Coronavirus Disease-19 Coronavirus Disease-19 Vaccine Coronavirus Disease 19 Vaccine Vaccine, Coronavirus Disease-19 COVID 19 Vaccine Vaccine, COVID 19 2019-nCoV Vaccine 2019 nCoV Vaccine Vaccine, 2019-nCoV 2019 Novel Coronavirus Vaccines 2019 Novel Coronavirus Vaccine 2019-nCoV Vaccines 2019 nCoV Vaccines Vaccines, 2019-nCoV SARS Coronavirus 2 Vaccines SARS2 Vaccine Vaccine, SARS2)) AND (hesitancy)

\section{Supplementary Files}

This is a list of supplementary files associated with this preprint. Click to download.

- PRISMAPchecklistforCOVID19Hesitancy.docx 